\title{
Ultrasonic inspection of a welded stainless steel pipe to evaluate residual stresses through thickness
}

\author{
Yashar Javadi ${ }^{1}$ \\ ${ }^{1}$ Department of Mechanical Engineering, Islamic Azad University-Semnan Branch, Semnan, Iran. \\ (Corresponding author's e-mail: yasharejavadi@yahoo.com; Tel: +98 9124402303, Fax: +98 2313354030.$)$
}

Hamed Salimi Pirzaman²

2Department of Mechanical Engineering, Amirkabir University of Technology, Tehran, Iran.

Mohammadreza Hadizadeh Raeisi ${ }^{3}$

${ }^{3}$ Department of Mechanical Engineering, Amirkabir University of Technology, Tehran, Iran.

Mehdi Ahmadi Najafabadi ${ }^{4}$

${ }^{4}$ Department of Mechanical Engineering, Amirkabir University of Technology, Tehran, Iran.

Abstract

This study investigates ultrasonic method in axial and hoop stress measurement through thickness of an austenitic stainless steel pipe. Longitudinal critically refracted $\left(L_{C R}\right)$ waves are employed to measure the welding residual stresses while outer and inner surfaces of the pipe are inspected by using different frequency range of ultrasonic transducers. The acoustoelastic constant is measured on a plate with the same material and thickness of the investigated pipe to keep the pipe intact. Welding process of the pipe is simulated by a 3D finite element (FE) model which is validated by hole-drilling method performed on 25 points. The residual stresses calculated by FE simulation are then compared with those obtained from the ultrasonic measurement while a good agreement is observed. It is demonstrated that the residual stresses through thickness of the stainless steel pipe can be evaluated by combining $F E$ and $L_{C R}$ method (known as $F E L_{C R}$ method).

Keywords: Finite Element Welding Simulation; Ultrasonic Stress Measurement; Through Thickness Stress Measurement; Longitudinal critically refracted waves; Acoustoelastic Constant.

\section{Introduction}

Residual stresses analysis is an essential stage in the design of mechanical equipments and in the evaluation of their reliability under real service conditions. The welding residual stresses, which are formed as the result of differential contractions during the weld solidification, might lead to a severe reduction in the fatigue strength of welded structures.

The residual stresses are measured by Olabi and Hashmi [1] where it has been concluded that the post-weld heattreatment lead to reducing residual stresses by about $70 \%$. The welding residual stresses are also evaluated by them in different works [2-5] to study the influence of various parameters on the stresses of welded structures manufactured from low carbon steel and stainless steel. 
Non-destructive measurement of residual stress is useful to optimize the structures' design and control their mechanical strength. One of the promising directions in the development of non-destructive techniques for residual stresses measurement is the application of ultrasound [6]. Ultrasonic method is a nondestructive way for stress measurement which is not limited by the material type and thickness. Longitudinal critically refracted $\left(L_{C R}\right)$ waves are widely used as a stress measurement technique in comparison with the other types of the ultrasonic waves. The technique is based on the acoustoelastic effect, according to which the velocity of elastic wave propagated in solids depends on the mechanical stress.

Further comprehensive discussion on ultrasonic nondestructive stress measurement is available from a number of sources, remarkably Thompson et al [7] and Schneider [8]. Applications of the $L_{C R}$ technique in the stress evaluation are given in a number of studies, particularly Santos and Bray [9], Bray and Tang [10] or Bray [11]. Tanala et al [12] explained a combination of subsurface longitudinal and Rayleigh waves to determine the welding stresses near surface. Employing the subsurface longitudinal waves in rail stress measurement has been studied by Eagle and Bray [13]. Palanichamy et al [14] measured the residual stresses in austenitic stainless steel weld joints by using ultrasonic technique. However, the ultrasonic stress measurement through thickness of a stainless steel pipe is not considered in the previous studies.

The main objective of this research is feasibility study for nondestructive stress measurement through thickness of an austenitic stainless steel pipe. The ultrasonic $L_{C R}$ waves are employed to measure the welding residual stresses. The investigated pipe thickness is greater than the penetration depths measured for ultrasonic transducers employed in this study. Hence, the pipe is ultrasonically inspected from both sides by using separate experimental setup prepared for inner and outer surface inspection. The acoustoelastic constant measurement is an inevitable stage in the process of ultrasonic stress evaluation. The acoustoelastic measurement is usually accomplished through the tensile test which needs cutting the tested material to extract tensile test specimens. However, the cutting process on the tested pipe is not considered in this study to complete ultrasonic measurement nondestructively. Instead, a welded plate with the same material and thickness of the investigated pipe is used to extract tensile test samples. The measured acoustoelastic constant of the plate along with the time of flight (TOF) of the $L_{C R}$ wave measured on the pipe, are employed for ultrasonic stress measurement. Four different series of transducers are utilized to measure the TOF of the $L_{C R}$ wave in different depths of the pipe thickness while their nominal frequencies are $5 \mathrm{MHz}, 4 \mathrm{MHz}, 2 \mathrm{MHz}$ and $1 \mathrm{MHz}$. Using this experimental configuration leads to a comprehensive knowledge of residual stress through thickness of the pipe which is nondestructively achieved. A 3D finite element model, which is validated by hole-drilling method performed on 25 points, is also employed to verify the ultrasonic results. The residual stresses calculated by FE simulation are then compared with those obtained from the ultrasonic measurement while a good agreement is observed. The combination of finite element and $L_{C R}$ method (known as $F E L_{C R}$ by Javadi et al [15]) is confirmed to be able to evaluate the pipe residual stresses through the thickness.

\section{Theoretical Background}

\section{1. $L_{C R}$ method}

Different ultrasonic configurations can be employed for residual stresses measurements by $L_{C R}$ technique. As a common experimental setup, longitudinal waves are propagated at the first critical angle by a transmitter transducer and then travel parallel the tested material surface and finally are detected by a receiver transducer. The residual stress in a subsurface layer is measurable while the depth of layer is related to the ultrasonic wave-length, often exceeding a few millimetres.

The relation between measured travel-time change of $L_{C R}$ wave and the corresponding uniaxial stress is derived by Egle and Bray [13] to be:

$$
\Delta \sigma=\frac{E}{L t_{0}}\left(t-t_{0}-\Delta t_{T}\right)
$$

where $\Delta \sigma$ is stress change, $E$ is the elastic modulus and $L$ is the acoustoelastic constant for longitudinal waves propagating in the direction of the applied stress field. Also, $t$ is the experimental travel-time which would be measured on the welded structure that is being evaluated; $t_{0}$ is travel-time for a homogeneous, isotropic, stress-free sample at the room temperature and $\Delta t_{T}$ is temperature difference effect between the measurement temperature and room temperature on the travel-time. With knowledge of the weld induced change in travel-time and the measured acoustoelastic constant, the stress change produced by the weld may be calculated. 


\subsection{Finite Element Welding Simulation}

Welding simulation by using the Finite Element (FE) method has become a popular tool for the prediction of welding residual stresses and deformations. Earlier studies of the welding simulation, due to weakness in the computational capabilities of the past computers, were limited to two-dimensional modeling. However, it is recently possible to employ 3D modeling of the welding process while good agreements have been observed between the numerical predictions and experimental results [16].

Numerical analysis of the welding residual stresses needs, to take account of the mechanical properties of welds, which is sensitive to the coupling among heat transfer, microstructure evolution and thermal stress analysis. The phenomena involved in the heat input such as arc, material interactions, as well as, fluid dynamics in the weld pool are not exactly described. From the thermo-mechanical point of view, the heat input can be considered as a volumetric or surfaced energy distribution, and the fluid flow effect, which leads to homogenize the temperature in the molten area, can be taken into account by rising the thermal conductivity over the melting temperature. Heat transfers in solids are described by the heat equation as following:

$\rho \frac{d H}{d t}-\operatorname{div}(k \nabla T)-Q=0$

$$
k \nabla T . n=q(T, t) \quad \text { on } \partial \Omega_{q}
$$

$$
T=T_{p}(t) \quad \text { on } \partial \Omega_{t}
$$

In Equation (2-4), $\rho, k, H, Q$ and $T$ are density, thermal conductivity, enthalpy, internal heat source and temperature respectively. In Equation (3), $\mathrm{n}$ is the outward normal vector of domain $\partial \Omega$ and $q$ is the heat flux density that can rely on temperature and time to model convective heat exchanges on the surface and $T_{p}$ is a prescribed temperature.

The heat input is represented by an internal heat source. In this study, the double ellipsoid heat source pattern (Fig. 1) presented by Goldak et al. [17] is employed to simulate the heat source in FE model. The front half of the heat source is quadrant of one ellipsoidal source while the rear half is the quadrant of another ellipsoid.

The Goldak equations for the front and rear heat source are represented by the Equation (5) and Equation (6) respectively:

$$
\begin{aligned}
& q_{f}(x, y, z)=\frac{6 \sqrt{3} f_{f} Q}{a b c_{f} \pi^{3 / 2}} e^{\left(-3 x^{2} / a^{2}\right)} e^{\left(-3 y^{2} / b^{2}\right)} e^{\left(-3 z^{2} / c^{2}\right)} \\
& q_{r}(x, y, z)=\frac{6 \sqrt{3} f_{r} Q}{a b c_{r} \pi^{3 / 2}} e^{\left(-3 x^{2} / a^{2}\right)} e^{\left(-3 y^{2} / b^{2}\right)} e^{\left(-3 z^{2} / c^{2}\right)}
\end{aligned}
$$

In Equation (5-6), $x, y$ and $z$ are the local coordinates of the double ellipsoid model aligned with the welded structure; $f_{f}$ and $f_{r}$ are parameters which give the fraction of the heat deposited in the front and rear parts respectively. $Q$ is the welding energy which is calculated by knowing the welding voltage, current and the arc efficiency. The parameter $a$ is one-half the width of melted zone; $b$ is depth of the melted zone; $c_{f}$ and $c_{r}$ are the front and behind section dimensions of the heat source respectively. The front section dimension of the heat source $\left(c_{f}\right)$ is assumed equal to $a$ while the rear section $\left(c_{r}\right)$ is equal to $4 \times a$. The relations $2 /\left(1+c_{r} / c_{f}\right)$ and $2 /\left(1+c_{f} / c_{r}\right)$ are employed to calculate the $f_{f}$ and $f_{r}$ respectively. The moving heat source is modelled by a user subroutine in the ANSYS commercial software.

Material modeling has always been a difficult issue in the welding simulation according to the lack of material data at elevated temperatures. Some simplifications and approximations are normally introduced to overcome this problem. These simplifications are needed due to both lack of data and numerical difficulties when trying to model the actual hightemperature properties of the material [18]. The elevated temperature properties of 304L stainless steel used in the finite element simulation is extracted from X.K.Zhu et al [19].

The problem is formulated as a successively coupled thermal stress analysis. First, a nonlinear thermal analysis is performed to calculate the temperature history of the entire domain. Then, the results of the thermal analysis are applied as a thermal body load in a nonlinear mechanical analysis determining residual stress and distortion. The finite element (FE) models for both thermal and structural analysis are the same. The general-purposed FE program ANSYS is used for the analysis. A full Newton-Raphson iterative solution technique with direct sparse matrix solver is employed for obtaining a 
solution. During the thermal analysis, the temperature and the temperature dependent material properties alter very rapidly. Thus, the full Newton-Raphson technique with using modified material properties is believed to give more precise results.

A conventional technique named "Element Birth and Death" is used for modelling of the deposited weld. A complete FE model is generated in the start of the analysis. However, all elements representing the deposited weld except elements for the tack welds are deactivated by assigning them a very low stiffness. During the thermal analysis, all the nodes of deactivated elements (excluding those shared with the base metal) are also fixed at room temperature till the birth of the respective elements. Deactivated elements are reactivated sequentially when they come under the effect of the welding torch. Here, eight-noded-brick elements with linear shape functions are used in the FE modeling.

A meshing investigation is done to find the most proper mesh according to the mesh size and accuracy of the FE results. The basic mesh size is shown in Fig. 2, while some finer and coarser meshing models are studied and the results are compared with the experimental measurements. Selecting the most effective mesh size leads to the accurate results along with time-consuming calculations. Only one side of the welded pipes is modeled with the symmetry assumption.

\section{Experimental Procedures}

\subsection{Sample Description}

Two $500 \mathrm{~mm}$ length pipes made of stainless steel (304L) are welded while outside diameter and thickness is equal to 320 $\mathrm{mm}$ and $10 \mathrm{~mm}$ respectively. Two $600 \times 250 \times 10 \mathrm{~mm}$ plates are also welded to be employed for acoustoelastic constant measurement. Material and thickness of the welded pipe and plate are the same. The weld reinforcements are removed by $30000 \mathrm{rpm}$ hand grinder to facilitate ultrasonic measurements. However, temperature during the grinding process is kept less than $50^{\circ} \mathrm{C}$ to prevent generation of thermal stresses.

\subsection{Measurement device}

The measurement device, shown in Fig. 3, includes an ultrasonic box, computer and time of flight (TOF) measuring unit. The ultrasonic box is a $100 \mathrm{MHz}$ ultrasonic testing device which has synchronization between the pulser signal and the internal clock, which controls the $A / D$ converter. This allows very precise measurements of the time of flight - better than $1 \mathrm{~ns}$, however a MATLAB subroutine is employed to increase the resolution to $0.1 \mathrm{~ns}$. TOF measuring element includes three normal transducers assembled on an integrated wedge to measure the time of flight. A poly methyl methacrylate (PMMA) material, under the trademark Plexiglas, is cut by laser to construct the wedge. Five types of wedges, shown in Fig. 4 , are needed to measure the TOF on the plate, pipe outer and inner surface in axial and hoop directions. A three-probe arrangement is utilized, with one sender and two receivers in order to eliminate the effects of environment temperature on the travel time. Twelve transducers in four different frequencies are used while their nominal frequencies are $1 \mathrm{MHz}, 2$ $\mathrm{MHz}, 4 \mathrm{MHz}$ and $5 \mathrm{MHz}$. Three normal transducers with the same frequency are assembled in each wedge where the diameter of the piezoelectric elements is $6 \mathrm{~mm}$. Moving step of TOF measuring unit is equal to $2 \mathrm{~mm}$ for the points near and on the melted zone (MZ) while it is changed from $10 \mathrm{~mm}$ to $40 \mathrm{~mm}$ far away the weld. The TOF is measured three times for each point and the average data is calculated. The path should be scanned four times by using the four different frequencies of the transducers.

\subsection{Determination of $L_{C R}$ Penetration Depth}

When the $L_{C R}$ technique is applied to an application with limited wall thickness, the penetration depth of the $L_{C R}$ wave is expected to be a function of frequency. However, there is no definite relation between the $L_{C R}$ depth and frequency. Hence, the $L_{C R}$ depth should be measured experimentally. A variable depth groove is cut in a pipe, with the same material and thickness of the investigated sample, to produce a barrier to physically prevent the $L_{C R}$ wave from reaching the receiver transducer. It is found that a $1 \mathrm{~mm}$ depth groove could completely prevent a $5 \mathrm{MHz} L_{C R}$ wave to pass, which indicates that the penetration depth of such a $L_{C R}$ wave is $1 \mathrm{~mm}$. Similarly, the penetration depth of $4 \mathrm{MHz}, 2 \mathrm{MHz}$ and 1 $\mathrm{MHz} L_{C R}$ wave is measured $1.5 \mathrm{~mm}, 3.5 \mathrm{~mm}$ and $6 \mathrm{~mm}$ respectively.

\subsection{Acoustoelastic constant evaluation of the Plate}

To evaluate the acoustoelastic constant $(L)$, the tensile test samples are taken from both sides of the plate. Rectangular tensile test specimens are extracted from parent material (PM), melted zone (MZ) and heat affected zone (HAZ) separately. Metallographic analysis of the weld shows that the heat affected zone (HAZ) is not large enough to extract tensile test sample. Therefore, samples are prepared from tensile test specimens, extracted from the parent material, to reproduce microstructure of HAZ by means of heat treatment. Each sample has experienced different annealing temperature, annealing time, cooling rate and cooling environment. Since no microstructure phase change occurred in the HAZ during 
the welding process, the austenite grain size in the microstructure of the sample is considered as a criterion in the reproduction of $\mathrm{HAZ}$ microstructure. From this point, the best agreement is found for the sample annealed at $1200^{\circ} \mathrm{C}$ for 7 min followed by an air cooling. The grain size of HAZ and simulated sample are close to the G5.5 according to the ASTM: E112 (standard test methods for determining average grain size). To evaluate the residual stress according to Eq. (1), the value $t_{0}$ is measured directly from the stress-free samples. The acoustoelastic constant $(L)$ is then deduced experimentally from a uniaxial loading calibration test.

\section{Results and Discussion}

\subsection{Acoustoelastic Constant of the Plate}

According to the results of tensile test along with calculations based on Eq. (1), the acoustoelastic constant $(L)$ related to the plate is measured equal to $1.839,2.102$ and 2.452 in the HAZ, PM and MZ respectively. The measured acoustoelastic constant of the plate is assumed same as the pipe constant and would be used in residual stresses calculations. The measurements show that the HAZ constant is less than the PM whereas the maximum is the MZ constant. This behavior of acoustoelastic constant was also reported by Javadi et al [15].

\subsection{Outer Surface Inspection of the Pipe}

In this study, a 3D finite element analysis is used to predict residual stresses through the pipe thickness. Since the thickness of the pipe is equal to $10 \mathrm{~mm}$ and maximum of penetration depth is measured $6 \mathrm{~mm}$ related to $1 \mathrm{MHz}$ transducer, it is necessary to inspect the pipe from both sides. It means that, outer and inner surface inspections are needed to reach a complete knowledge of the residual stresses through thickness.

The results of the finite element method are shown in Fig. 5 and Fig. 6 corresponds to the hoop and axial residual stress respectively. Since the results are related to the outer surface, the depths shown in the figures are from the outside of the pipe, i.e. the $0 \mathrm{~mm}$ shows the outer surface and $10 \mathrm{~mm}$ shows the inner surface. Furthermore, these depths are selected according to the measured penetration depth of the ultrasonic transducers.

The FE simulation of weld shows that the hoop residual stress is tensile in center of the weld whereas it converts to compressive stress near the HAZ. The axial stress is compressive in the weld centerline which shows a stress distribution in the opposite direction of hoop stress on the outer surface of the pipe. The distribution of hoop and axial residual stress is comparable with the welding logic and the results of previous studies (for example the FE results of dissimilar pipe analyzed by Javadi et al [20]).

A comparison between FE simulation results of inner and outer surface of the pipe shows that the hoop stress behavior of the pipe outer surface is similar to the inner surface despite the fact that the axial stress considerably differs through the thickness. The hoop stress changes from 58.17 MPa to 340.08 MPa by moving from the outer to inner surface while the axial stress converts from -239.12 MPa to 247.13 MPa in center of the weld. A similar hoop and axial stress distribution through thickness of pipes was reported by some of the previous studies [21-23].

It should be noted that, the wave speed for $L_{C R}$ waves is affected by the average stress in a layer which may be a few millimeters thick [14]. For example, the $1 \mathrm{MHz} L_{C R}$ wave travels in $6 \mathrm{~mm}$ beneath the surface and gives the average of residual stress in this zone. Therefore, the FE residual stresses for all the nodes located in $6 \mathrm{~mm}$ under the surface are extracted and their averages are calculated to compare with measurements performed by the $1 \mathrm{MHz} L_{C R}$ wave. It means that, the results shown in Fig. 5 and Fig. 6 cannot be compared with the ultrasonic results because they are exact results. Therefore, the averages of FE residual stresses are calculated according to the penetration depth of the transducers which are shown in Fig. 7.

To compare FE results with ultrasonic measurements, it is needed to verify the FE model with the experimental results. The residual stress of 25 points is measured by the hole-drilling method in axial and circumferential direction while the results are compared with the FE analysis according to Fig. 8 and Table 1. The average results of FE residual stress in $2 \mathrm{~mm}$ depth are compared with those of hole-drilling method because this method also gives the average of residual stress measured along the $2 \mathrm{~mm}$ depth hole. The hole-drilling method is performed based on the characterizations described in ASTM: E837. The comparison between FE analysis and hole-drilling results show good agreement which validate the FE welding simulation.

The results of ultrasonic measurements are shown in Fig. 9-Fig. 12 according to the frequency of ultrasonic transducer used to measure the TOF. All of the results of ultrasonic measurements are compared with finite element analysis showing an acceptable agreement. The disagreement between FE and ultrasonic, listed in Table 2, is differed according to the ultrasonic test frequency and tested zone. 
According to the results of Table 2, the ultrasonic accuracy in PM is higher than MZ and HAZ. Despite of the same material and thickness of the plate and pipe, there are some differences in the welding specifications which lead to relatively high error in acoustoelastic constant evaluation of $\mathrm{MZ}$ and $\mathrm{HAZ}$.

Comparison between different test frequencies shows that the $L_{C R}$ waves work more accurately by employing the lower frequency transducers. It means that the disagreements between the $\mathrm{FE}$ and ultrasonic measurement performed by $5 \mathrm{MHz}$ transducer is higher than those obtained by $4 \mathrm{MHz}$ and the minimum occurs at $1 \mathrm{MHz}$. Higher error of higher frequencies can be justified by the fact that the low frequency transducers produce sharp and more powerful echo in the receiver than high frequency transducers. Therefore it is necessary to increase the "Gain" by using higher frequencies which will lead to less resolution and higher measuring error in the TOF. The less precise measurement performed by high frequency waves, was also reported by Javadi et al [15].

However, the disagreement between the FE and ultrasonic results does not exceed than $\pm 40 \mathrm{MPa}$ which is an acceptable error for a nondestructive stress measurement method.

\subsection{Inner Surface Inspection of the Pipe}

The results of the finite element method are shown in Fig. 13 and Fig. 14 for different depths corresponds to the hoop and axial residual stress respectively. Since the results are related to the inner surface, the depths shown in the figures are from the inside of the pipe, i.e. the $0 \mathrm{~mm}$ shows the inner surface and $10 \mathrm{~mm}$ shows the outer surface. The averages of FE residual stresses are also calculated, shown in Fig. 15, to be comparable with the ultrasonic measurements.

The results of ultrasonic measurements are shown in Fig. 16-Fig. 19 according to the frequency of ultrasonic transducer used to measure the TOF. All of the results of ultrasonic measurements are compared with finite element analysis showing an acceptable agreement while the disagreements between FE and ultrasonic are listed in Table 3.

The disagreement results of inner surface inspection are similar to those obtained from the outer surface measurements. However, in both of the inspections the disagreement between the FE and ultrasonic results does not exceed than \pm 40 $\mathrm{MPa}$ which confirms the capability of $F E L_{C R}$ method (which is the combination of finite element welding simulation and ultrasonic stress measurement by $L_{C R}$ waves [15]) to evaluate welding residual stress through the thickness.

\section{Conclusion}

The main goal of this paper is ultrasonic evaluation of hoop and axial residual stresses in a stainless steel pipe through the thickness. The ultrasonic measurements are compared with residual stresses obtained from a FE analysis validated by holedrilling method. According to the achieved results, it can be concluded that:

1- The hoop residual stress of the inner and outer surface is tensile in the weld centerline while the axial stress is notably differs through the thickness of the pipe.

2- The $L_{C R}$ ultrasonic method measures the average of stresses in determined penetration depth of transducers. Therefore, if measuring the stress in an exact depth is needed, the ultrasonic method is not recommended.

3- Since the penetration depths of the ultrasonic transducers are limited to a few millimeters, both sides measurement of thick materials is recommended.

4- A little difference in the welding specifications of pipe with the plate used to measure acoustoelastic constant, lead to relatively high error in stress evaluation of MZ and HAZ.

5- The average results of FE residual stress in $2 \mathrm{~mm}$ from the surface are in good agreement with those of holedrilling method.

6- Ultrasonic measurements of hoop and axial residual stresses are compared with finite element analysis and show an acceptable agreement.

7- The disagreement between the FE and ultrasonic results is increased by using high frequency transducers.

8- The $F E L_{C R}$ method can evaluate welding residual stress through the thickness of stainless steel pipe.

\section{References}

[1] Olabi AG, Hashmi MSJ. The effect of post-weld heat-treatment on mechanical-properties and residual-stresses mapping in welded structural steel. Journal of Materials Processing Technology 1995; 55: 117-22.

[2] Olabi AG, Hashmi MSJ. The Microstructure and Mechanical Properties of Low Carbon Steel Welded Components After the Application of PWHTs. Journal of Materials Processing Technology 1996; 56:88-97. 
[3] Olabi AG, Hashmi MSJ. Stress Relief Procedures for Low Carbon Steel (1020) Welded Components. Journal of Materials Processing Technology 1996; 56:552-62.

[4] Olabi AG, Hashmi MSJ. Effects of the stress-relief conditions on a martensite stainless-steel welded component. Journal of Materials Processing Technology 1998; 77: 216-25.

[5] Olabi AG, Casalino G, Benyounis KY, Rotondo A. Minimisation of the residual stress in the heat affected zone by means of numerical methods. Materials and Design 2007; 28: 2295-302.

[6] Rossini NS, Dassisti M, Benyounis KY, Olabi AG. Methods of measuring residual stresses in components. J Materials \& Design 2012; 35:572-88.

[7] Thompson RB, Lu WY, Clark Jr AV. Handbook of Measurement of Residual Stress. In: Lu J, James M, Roy G, editors. Bethel: Society for Experimental Stress Analysis; 1996, p. 149-78.

[8] Schneider E. Structural and Residual Stress Analysis by Nondestructive Methods. In: Hauk V, editor. Amsterdam: Elsevier; 1997, p. 522-63.

[9] Santos A, Bray DE. Ultrasonic Stress Measurement Using PC Based and Commercial Flaw Detectors. Rev Sci Instrum 2000; 71: 3464-69.

[10] Bray DE, Tang W. Subsurface Stress Evaluation in Steel Plates and Bars with the $L_{C R}$ Ultrasonic Wave. Nuclear Engineering and Design 2001; 207: 231-40.

[11] Bray DE. Ultrasonic Stress Measurement in Pressure Vessels, Piping and Welds. Journal of Pressure Vessel Technology 2002; 124: 326-35.

[12] Tanala E, Bourse G, Fremiot M, De Belleval JF. Determination of near surface residual stresses on welded joints using ultrasonic method. NDT\&E International 1995; 28: 83-8.

[13] Egle DM, Bray DE. Measurement of Acoustoelastic and Third-Order Elastic Constants for Rail Steel. J Acoust Soc Am 1976; 60: 741-44.

[14] Palanichamy $P$, Vasudevan $M$, Jayakumar T. Measurement of residual stresses in austenitic stainless steel weld joints using ultrasonic technique. Science and Technology of Welding and Joining 2009; 14: 166-71.

[15] Javadi Y, Akhlaghi M, Ahmadi Najafabadi M. Using Finite Element and Ultrasonic Method to Evaluate Welding Longitudinal Residual Stress through the Thickness in Austenitic Stainless Steel Plates. J Materials \& Design 2013; 45: 628-42.

[16] Sattari-Far I, Javadi Y. Influence of welding sequence on welding distortions in pipes. Int J of Pressure Vessels and Piping 2008; 85: 265-74.

[17] Goldak J, Bibby M. Computational Thermal Analysis of Welds. Modeling of Casting and Welding Processes 1988; 4: 153-66.

[18] Lindgren LE. Finite element modelling and simulation of welding part 2: improved material modeling. J Thermal Stress 2001; 24:195-231.

[19] Zhu XK, Chao YJ. Numerical simulation of transient temperature and residual stresses in friction stir welding of 304L stainless steel. Journal of Materials Processing Technology 2004; 146: 263-72.

[20] Javadi Y, Ahmadi Najafabadi M. Comparison between contact and immersion ultrasonic method to evaluate welding residual stresses of dissimilar joints. J Materials \& Design 2013; 47: 473-82.

[21] Deng D, Murakawa H. Numerical simulation of temperature field and residual stress in multi-pass welds in stainless steel pipe and comparison with experimental measurements. Computational Materials Science 2006; 37:269-77.

[22] Malik AM, Qureshi EM, Ullah DN, Iqbal K. Analysis of circumferentially arc welded thin-walled cylinders to investigate the residual stress fields. Thin-Walled Structures 2008; 46: 1391- 401.

[23] Lee $\mathrm{CH}$, Chang KH. Prediction of residual stresses in high strength carbon steel pipe weld considering solid-state phase transformation effects. Computers and Structures 2011; 89: 256-65. 


\section{List of Table Captions}

Table 1. Comparison of FE and Hole-Drilling results in the Circumferential Direction corresponding to the Pipe Outer Surface Inspection

Table 2. Disagreement between FE and ultrasonic results (Pipe Outer Surface Inspection)

Table 3. Disagreement between FE and ultrasonic results (Pipe Inner Surface Inspection) 


\section{List of figures caption}

Fig. 1. Double ellipsoid heat source configuration used for FE simulation of welding heat source [17]

Fig. 2. Basic FE model of the Pipe

Fig. 3. Measurement Devices for TOF measurement of the Pipe (a,b: Inspection of the Pipe Outer Surface; $c$ : Inspection of the Pipe Inner Surface)

Fig. 4. Wedge Design

Fig. 5. FE Hoop Residual Stresses corresponding to the Pipe Outer Surface Inspection

$$
\text { (a: } X=0-500 \mathrm{~mm} ; \mathrm{b}: \mathrm{X}=0-100 \mathrm{~mm} \text { ) }
$$

Fig. 6. FE Axial Residual Stresses corresponding to the Pipe Outer Surface Inspection

$$
\text { (a: } X=0-500 \mathrm{~mm} ; \mathrm{b}: X=0-100 \mathrm{~mm} \text { ) }
$$

Fig. 7. Average of a) Hoop and b) Axial Residual Stresses obtained from FE analysis corresponding to the Pipe Outer Surface Inspection

Fig. 8. Comparison of FE and Hole-Drilling results in the Axial Direction corresponding to the a) Hoop and b) Axial Residual Stresses (Pipe Outer Surface Inspection)

Fig. 9. Comparison of FE and Ultrasonic results performed by $5 \mathrm{MHz}$ Transducer corresponding to the a) Hoop and b) Axial Residual Stresses (Pipe Outer Surface Inspection)

Fig. 10. Comparison of FE and Ultrasonic results performed by $4 \mathrm{MHz}$ Transducer corresponding to the a) Hoop and b) Axial Residual Stresses (Pipe Outer Surface Inspection)

Fig. 11. Comparison of FE and Ultrasonic results performed by $2 \mathrm{MHz}$ Transducer corresponding to the a) Hoop and b) Axial Residual Stresses (Pipe Outer Surface Inspection)

Fig. 12. Comparison of FE and Ultrasonic results performed by $1 \mathrm{MHz}$ Transducer corresponding to the a) Hoop and b) Axial Residual Stresses (Pipe Outer Surface Inspection)

Fig. 13. FE Hoop Residual Stresses corresponding to the Pipe Inner Surface Inspection

$$
\text { (a: } X=0-500 \mathrm{~mm} ; \mathrm{b}: \mathrm{X}=0-100 \mathrm{~mm} \text { ) }
$$

Fig. 14. FE Axial Residual Stresses corresponding to the Pipe Inner Surface Inspection

$$
\text { ( } a: X=0-500 \mathrm{~mm} ; \mathrm{b}: X=0-100 \mathrm{~mm} \text { ) }
$$


Fig. 15. Average of a) Hoop and b) Axial Residual Stresses obtained from FE analysis corresponding to the Pipe Inner Surface Inspection

Fig. 16. Comparison of FE and Ultrasonic results performed by $5 \mathrm{MHz}$ Transducer corresponding to the a) Hoop and b) Axial Residual Stresses (Pipe Inner Surface Inspection)

Fig. 17. Comparison of FE and Ultrasonic results performed by $4 \mathrm{MHz}$ Transducer corresponding to the a) Hoop and b) Axial Residual Stresses (Pipe Inner Surface Inspection)

Fig. 18. Comparison of FE and Ultrasonic results performed by $2 \mathrm{MHz}$ Transducer corresponding to the a) Hoop and b) Axial Residual Stresses (Pipe Inner Surface Inspection)

Fig. 19. Comparison of FE and Ultrasonic results performed by $1 \mathrm{MHz}$ Transducer corresponding to the a) Hoop and b) Axial Residual Stresses (Pipe Inner Surface Inspection) 